\title{
Charge-exchange spectroscopy at the W7-AS stellarator employing a high-energy Li beam
}

\author{
H Ehmler ${ }^{1,3}$, J Baldzuhn ${ }^{1,3}$, K McCormick ${ }^{1}$, A Kreter ${ }^{2}$, T Klinger ${ }^{1,3}$ and \\ W7-AS Team \\ ${ }^{1}$ Max-Planck-Institut für Plasmaphysik, Boltzmannstrasse 2, D-85748 Garching, EURATOM \\ Association, Germany \\ 2 Institut für Plasmaphysik, Forschungszentrum Jülich GmbH, D-52425 Jülich, Germany \\ E-mail: ehmler@ipp.mpg.de
}

Received 10 September 2002

Published 16 December 2002

Online at stacks.iop.org/PPCF/45/53

\begin{abstract}
Combined measurements of ion density, temperature and poloidal rotation of impurity ions have been carried out in the gradient region of the Wendelstein 7-AS plasma, employing charge-exchange spectroscopy with the high-energy neutral $\mathrm{Li}$ beam and $\mathrm{C}^{6+} / \mathrm{C}^{5+}$ ions. The present state of the experimental technique is described. Results are obtained in discharges with high central ion temperatures of $1.3 \mathrm{keV}$, central electron densities of $7 \times 10^{19} \mathrm{~m}^{-3}$. Under these conditions the diagnostic covers a region of about $7 \mathrm{~cm}$ radial extent, corresponding to ion temperatures between 50 and $800 \mathrm{eV}$. Poloidal rotation velocities up to $(35 \pm 2.5) \mathrm{km} \mathrm{s}^{-1}$ were measured in a narrow shear layer at the plasma edge. The contribution of ion diamagnetic and $E \times B$ drift is discussed. The radial electric field is derived from a simplified radial force balance equation.
\end{abstract}

\section{Introduction}

Charge-exchange spectroscopy (CXS) in fusion plasmas [1] allows one to determine the concentrations, temperatures and fluid velocities of impurity ions. CXS is either applied to heating beams, or to specially designed neutral diagnostic beams [2], which are optimized for spatial resolution and CX-signal qualities. A diagnostic Li beam [3,4] provides a focused $(\approx 10 \mathrm{~mm}$ width), high-energy $(30-80 \mathrm{keV})$ neutral beam with negligible heating effect. It is operated in pulsed sequences of several milliseconds, which is necessary for the discrimination between the active $\mathrm{CX}$-signal and passive background radiation. From the light emission profile of the $\mathrm{Li}(2 \mathrm{p} \rightarrow 2 \mathrm{~s})$ transition, the local electron density and the population of the $\mathrm{Li}_{n l}$ levels up to $n=4$ is inferred [5]. Measurements of the temperature and density of $\mathrm{C}^{6+}$ ions by Libeam-CXS were carried out in proof-of-principle experiments [6-9]. The determination of $\mathrm{C}^{6+}$ poloidal rotation velocities has not yet been successful, mainly due to a poor signal-to-noise

3 Present address: IPP Teilinstitut Greifswald, Wendelsteinstrasse 1, D-17491 Greifswald, Germany. 
ratio. In this work, these problems could be solved by improving the optical throughput and detection efficiency of the diagnostic set-up.

\section{Experimental set-up}

Experiments were conducted on the Wendelstein 7-AS stellarator [10]. The charge-exchange reaction

$$
\mathrm{Li}^{0}+\mathrm{C}^{Z+} \longrightarrow \mathrm{Li}^{+}+\mathrm{C}^{(Z-1)+}+h v\left(n \rightarrow n^{\prime}\right)
$$

is employed for $Z=6\left(n=8 \rightarrow n^{\prime}=7\right.$ transition at $\left.\lambda_{0}=529.0 \mathrm{~nm}\right)$ and $Z=5$ $\left(n=7 \rightarrow n^{\prime}=6\right.$ transition at $\left.\lambda_{0}=494.5 \mathrm{~nm}\right)$ [6]. Figure 1 shows the experimental arrangement. The light emission at 14 radial positions along the beam line with $\Delta r \approx 0.6 \mathrm{~cm}$ radial spacing is picked up with optical fibre bundles via two lenses (not shown), covering a solid angle of $\Omega / 4 \pi \approx 3 \times 10^{-4}$. Each bundle consists of a $2 \times 4$ array of $400 \mu \mathrm{m}$ quartz fibres, imaging an area of $6 \times 12 \mathrm{~mm}$ of the beam. The fibres lead to the unit equipped with interferencefilters $(529,0.9 \mathrm{~nm}$ centre, bandwidth) and photomultipliers (PMs) (HAMAMATSU R928, quantum efficiency $15 \%)$. Because of spectral tolerances $( \pm 0.5 \mathrm{~nm})$, each filter is tilted by an angle $<5^{\circ}$ to correct the central wavelength. Up to five radial channels can be coupled alternatively to the entrance slit of a $0.75 \mathrm{~m}$ imaging Czerny-Turner spectrometer (JOBIN YVON 750M, $f=6$, grating $110 \times 110 \mathrm{~mm}^{2}, 1200$ grooves $\mathrm{mm}^{-1}$ blazed at $500 \mathrm{~nm}$ ). For that purpose, optical connectors $(5 \times 1 \mathrm{~mm}$ quartz fibres) were designed, fixed with one end to the entrance slit of the spectrometer. For a frequent spectral calibration a neon lamp is used with the spectral lines at $528.009,529.819$ and $530.476 \mathrm{~nm}$. To achieve a compromise between spectral resolution and light intensity, the entrance slit is tuned to $0.1 \mathrm{~mm}$. The resolution achieved is $\Delta \lambda_{\text {instr }}=0.05 \mathrm{~nm}$ (Gaussian width of Ne line). This corresponds to a Doppler-broadening of carbon ions with $50 \mathrm{eV}$ temperature (see below). A Peltier-cooled, back-illuminated charge coupled device (CCD) frame transfer camera (ROPER SCIENTIFIC MICROMAX:512BFT) with $512 \times 512$ pixels (dimension $6.7 \times 6.7 \mathrm{~mm}^{2}$ ) is employed. Spectra are recorded every $15 \mathrm{~ms}$, including a readout time of $6 \mathrm{~ms}$ for five binned strips. The CCD timing is synchronized to the beam chopper voltage and acquires subsequent frames with the beam turned either on or off to discriminate the active Li CX-signal from the passive background light. To improve

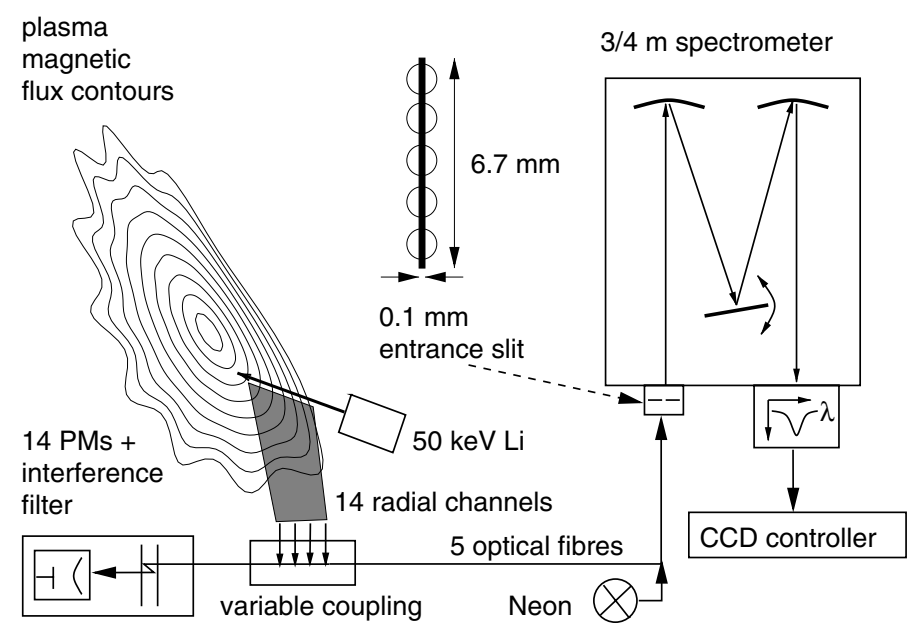

Figure 1. CXS set-up at the Wendelstein 7-AS stellarator. 
the signal-to-noise ratio, signals are time-averaged over typically $100-200 \mathrm{~ms}$ of stationary discharge conditions.

To demonstrate the capabilities of the diagnostic, discharges with strong active CX-signals were selected. A series of identical shots was carried out to achieve high ion temperatures (shots \#54233-54242). $2 \mathrm{MW}$ neutral beam injection and $1 \mathrm{MW}$ electron cyclotron resonance heating were applied in a pulse of $0.45 \mathrm{~s}$ duration. The magnetic configuration is such that the divertor modules limit the plasma at an effective radius of $15.5 \mathrm{~cm}$. General features of these discharges are discussed in $[11,12]$. Central values of electron density $n_{e}(0)=7.5 \times 10^{19} \mathrm{~m}^{-3}$, electron temperature $T_{e}(0)=1.5 \mathrm{keV}$ and ion temperature $T_{i}(0)=1.3 \mathrm{keV}$ were measured (cf figure 4). Under these conditions, the CX-signals are strong due to high Li beam penetration and large $\mathrm{C}^{6+}$ concentrations $\left(1-2 \%\right.$ of $\left.n_{e}\right)$.

Figure 2 shows spectra taken from two subsequent CCD frames at one radial position, where the ratio of active CX-signal to passive background is best. The distinct second line at $494.1 \mathrm{~nm}$ is probably due to emission from $\mathrm{B}^{1+}$ stemming from boronization of the vacuumvessel [14]. $\mathrm{B}^{5+}$ probably contributes to the measured $\mathrm{C}^{5+}$ signal because of the overlap of spectral lines which cannot be resolved. This is especially the case at inner radii, where carbon and boron are expected to be fully ionized. Contributions of $\mathrm{O}^{6+}$ to the measured $\mathrm{C}^{6+}$ signal can almost totally be excluded because of strong oxygen reduction by the boronization. The active to passive signal ratio is better for $\mathrm{C}^{6+}$ than for $\mathrm{C}^{5+}$ at most radial positions, but never exceeded $20 \%$. From the ratio of detected and total emitted CX photons at one radial position, an overall light gathering efficiency of $1.2 \times 10^{5} \mathrm{ph} \mathrm{s}^{-1} / 4.5 \times 10^{11} \mathrm{ph} \mathrm{s}^{-1}=2.8 \times 10^{-7}$ is calculated for the spectrometer. This agrees roughly with the estimated efficiency taking into account the solid angle, all optical transmissions, the throughput and the detection efficiency. For the PM system the overall efficiency is about a factor of 50 higher due to larger throughput. The noise of the recorded CX spectra arises mainly from photon statistics. Plasma fluctuations and the
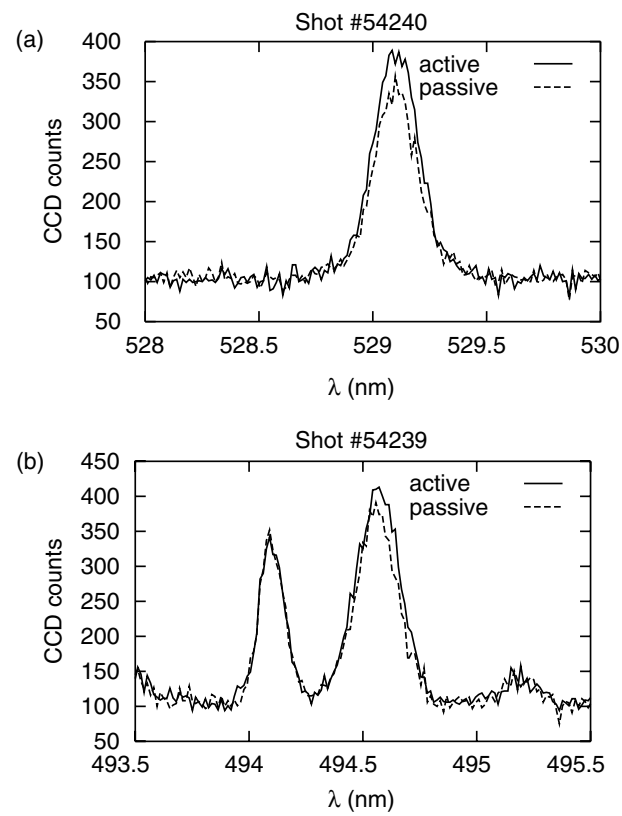

Figure 2. Spectra of subsequent CCD-frames at a radial position of $r=12.9 \mathrm{~cm}$ with $2.5 \mathrm{~mA}$ beam current. (a) $\mathrm{CX}$ with $\mathrm{C}^{6+}$ and (b) $\mathrm{CX}$ with $\mathrm{C}^{5+}$. 
dark current of the $\mathrm{CCD}$, which is cooled to $-15^{\circ} \mathrm{C}$, are of minor importance. To improve the signal-to-noise ratio for the evaluation of plasma parameters, CX spectra were time-averaged in the flat-top phase of the discharge from 0.2 to $0.45 \mathrm{~s}$ and averaged over identical shots.

\section{Plasma parameters measured by $\mathrm{Li}$ beam $\mathrm{CX}$}

\subsection{Ion temperature}

The CX spectra are fitted by a Gaussian

$$
I_{\mathrm{CX}}(\lambda)=I_{0} \exp \left[-\left(\frac{\lambda-\bar{\lambda}}{\Delta \lambda}\right)^{2}\right],
$$

where $\bar{\lambda}$ is the central wavelength and $\Delta \lambda$ the Gaussian width of the measured spectrum. Figure 3 shows measured spectra for $C^{6+}$ and $C^{5+}$ together with a best fit of equation (2). The statistical uncertainties from time averaging are indicated as error bars.

The ion temperature $T_{I}$ is given by

$$
T_{I}=\frac{M_{I}}{2 k_{\mathrm{B}}}\left(\frac{c}{\bar{\lambda}}\right)^{2}\left(\Delta \lambda^{2}-\Delta \lambda_{\text {instr }}^{2}\right),
$$

with the index $I$ denoting the species $\left(\mathrm{C}^{6+}\right.$ or $\left.\mathrm{C}^{5+}\right), M$ the mass, $k_{\mathrm{B}}$ the Boltzmann constant and $\Delta \lambda_{\text {instr }}$ the instrumental resolution. Fine structure splitting and the Zeeman effect cannot be resolved but lead to additional line broadening. In case of $\mathrm{C}^{6+}$, these effects are included via correction factors for the measured temperatures [7]. These corrections are less than $30 \%$ for ion temperatures above $100 \mathrm{eV}$. In case of $\mathrm{C}^{5+}$, no corrections were applied due to the lack of atomic data.

Figure 4 shows the plasma profiles of the shot series documented by different diagnostics, which are mapped onto effective radial coordinates. Ion temperatures of different ion species
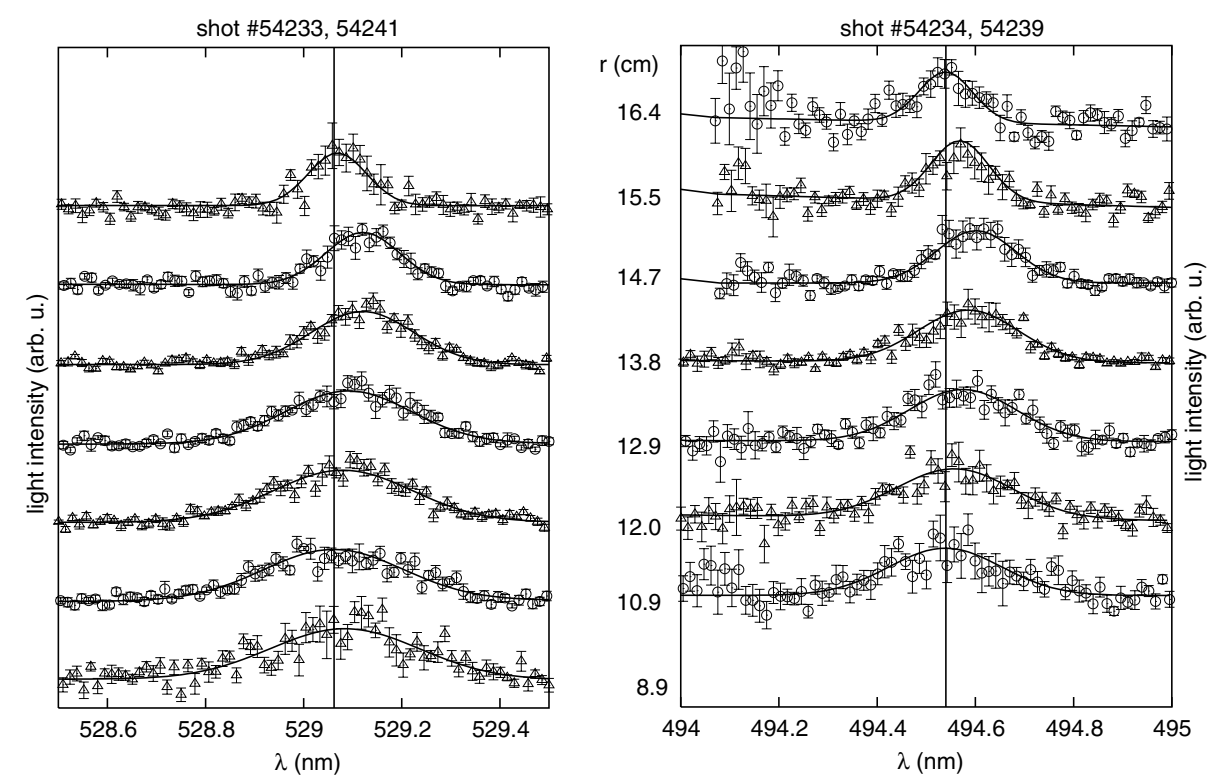

Figure 3. Time-averaged CX spectra at different radial positions. The non-Doppler shifted wavelength is indicated by a vertical line. Data are shown for $\mathrm{C}^{6+}$ (left) and $\mathrm{C}^{5+}$ (right). 
shot \#54233-54242
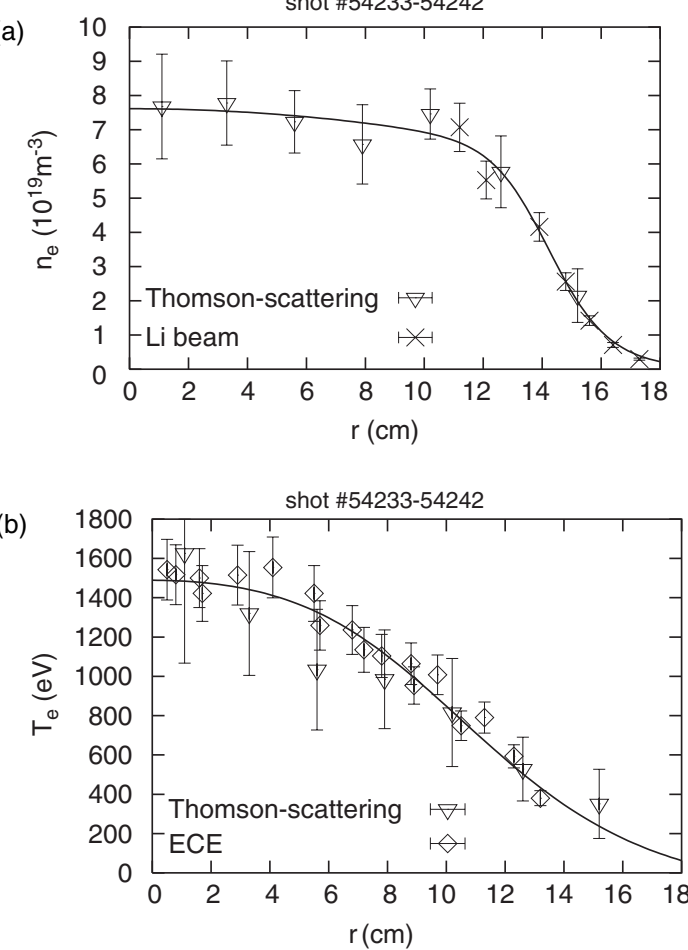

(c)

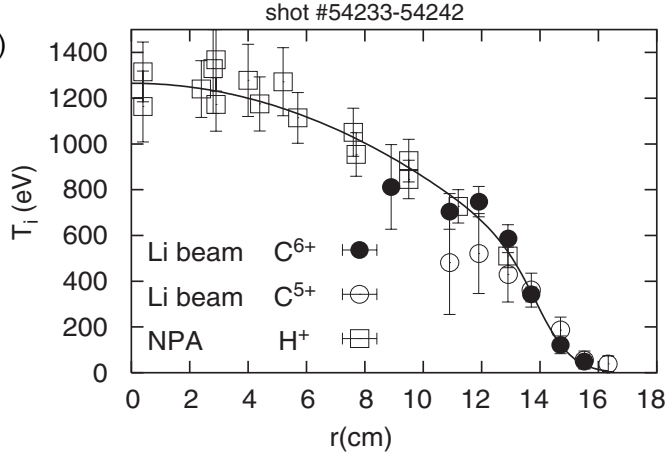

Figure 4. Radial profiles of $(a)$ electron density, $(b)$ electron temperature and $(c)$ ion temperature as obtained by Li beam diagnostic, Thomson scattering, electron cyclotron emission (ECE) and neutral particle analyzer (NPA) [11] as a function of effective plasma radius.

agree within their respective uncertainties. Therefore, it can be assumed that $T_{I}=T_{i}\left(i=\mathrm{H}^{+}\right.$ background plasma). The measured radial profiles of $n_{e}$ and $T_{i}$ are consistent among Li beam and other diagnostics.

\subsection{Impurity density}

The $\mathrm{C}^{6+}$ density is given by

$$
n_{\mathrm{C}^{6+}}=\mathrm{c}_{\mathrm{cal}} \frac{I_{\mathrm{CX}}}{\sum_{n, l} N_{\mathrm{Li}}^{n l} \sigma^{n l}},
$$


where $c_{\mathrm{cal}}$ is the calibration factor for the respective optical system, $I_{\mathrm{CX}}$ is the CX-signal intensity (PM signal if equation (5) is used, or sum of CCD counts if equation (6) is applied, respectively), $N_{\mathrm{Li}}^{n l}$ the relative population of the $\mathrm{Li}(n, l)$-level and $\sigma^{n l}$ the state-selective emission cross-section $\left(\sigma^{2 \mathrm{~s}}=1.12 \times 10^{-15} \mathrm{~cm}^{2}\right.$ and $\left.\sigma^{2 \mathrm{p}}=2.27 \times 10^{-15} \mathrm{~cm}^{2}\right)$ [13]. The contribution of Li levels higher than $n=2$ to the total cross-section is typically less than $5 \%[14]$.

For the PM system an absolute calibration for each radial position was obtained in situ by a beam-shaped tungsten lamp, which was inserted into the vessel replacing the Li beam. The calibration factor for the PM system is

$$
\mathrm{c}_{\mathrm{cal}}=\frac{4 \pi e \lambda_{0}}{h c} \frac{p_{\lambda} \Delta \lambda_{\mathrm{filter}} d}{J \eta \gamma I_{\mathrm{cal}}}
$$

where $p_{\lambda}=7.8 \times 10^{-3} \mathrm{~W} \mathrm{~m}^{-2} \mathrm{sr}^{-1}(\mathrm{~nm})^{-1}$ is the spectral emissivity, $I_{\text {cal }}$ the PM signal from the calibration lamp, $\Delta \lambda_{\text {filter }}=0.9 \mathrm{~nm}$ the bandwidth of the interference-filter, $d=12 \mathrm{~mm}$ the observed width of one optical channel, $J=1-3 \mathrm{~mA}$ the beam current, $\eta=0.75$ the neutralization efficiency and $\gamma=0.6$ the fraction of the beam observed. Due to uncertainties in cross-section data, drifts and ageing of optical transmissions, electronic equipment and beam properties (e.g. alignment, width and neutralization efficiency) the error in the calibration factors can be as large as $\pm 40 \%$.

When using the spectrometer, a much more reliable calibration of absolute $\mathrm{C}^{6+}$ densities is possible: the CX light at $529 \mathrm{~nm}$ and the $\mathrm{Li}_{2 \mathrm{p}}$ light at $671 \mathrm{~nm}$ is measured in two identical discharges with the spectrometer. The calibration factor $c_{\text {cal }}$ is then given by

$$
\mathrm{c}_{\mathrm{cal}}=\frac{s_{529 \mathrm{~nm}}^{671 \mathrm{~m}}}{\tau_{2 \mathrm{p}} v_{\mathrm{Li}}} \frac{N_{2 \mathrm{p}}}{I_{2 \mathrm{p}}} .
$$

Here, $s_{529 \mathrm{~nm}}^{671 \mathrm{~nm}}=0.42$ is the ratio of spectral sensivities of the entire optical system taken from technical specifications, $\tau_{2 p}=27 \mathrm{~ns}$ the lifetime of the $\mathrm{Li}(2 \mathrm{p} \rightarrow 2 \mathrm{~s})$ transition, $v_{\mathrm{Li}}=1174 \mathrm{~km} \mathrm{~s}^{-1}$ the beam velocity of $50 \mathrm{keV}^{7} \mathrm{Li}$ and $I_{2 \mathrm{p}}$ the sum of CCD counts of the $\mathrm{Li}_{2 \mathrm{p}}$ line. By this relative measurement, the absolute beam strength as well as the imaging properties cancel out. The resulting systematic error is mainly determined by the reliability of the used cross-section data. The density of $\mathrm{C}^{5+}$ could not be determined accurately due to the influence of boron and insufficient cross-section data.

Figure 5 shows radial profile measurements of the $\mathrm{C}^{6+}$ density recorded with both the PM system and the spectrometer. The agreement of both profiles is quite reasonable. The PM system has advantages given by the better photon statistics, but the calibration factors

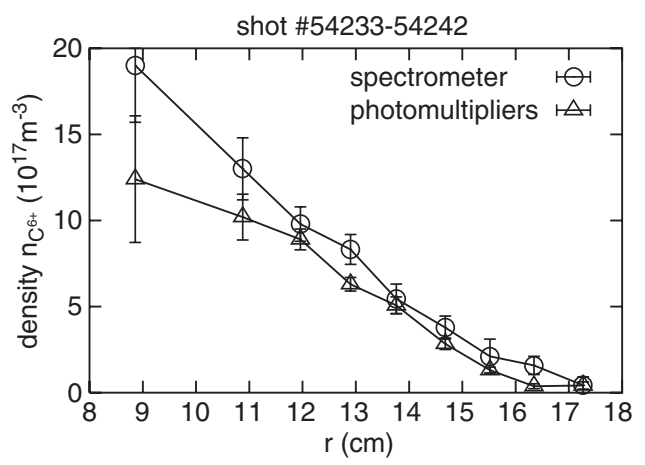

Figure 5. Impurity density $n_{\mathrm{C}^{6+}}$ measured by the PM system $(\triangle)$ and the spectrometer $(\mathrm{O})$. 
for each radial channel have a higher uncertainty than those for the spectrometer. The PM system is well suited for routinely measuring the carbon concentration as described in [14]. The spectrometer may be preferred for detailed comparisons of the impurity density profile with transport calculations, since the uncertainty in the relative calibration factors of different spatial channels is very low [15].

\subsection{Poloidal rotation and radial electric field}

The poloidal rotation velocity $v_{\theta}$ of the impurity ions moving on the magnetic flux surfaces leads to a Doppler shift of the observed central wavelength $\bar{\lambda}$ with respect to the non-shifted reference wavelength $\lambda_{0}$ :

$$
v_{\theta}=\frac{\bar{\lambda}-\lambda_{0}}{\lambda_{0}} c .
$$

The exact value of $\lambda_{0}$ is determined by the spectral overlap of fine structure components with different intensity. In case of $\mathrm{CX}$ with $\mathrm{C}^{6+}$, the line splitting covers a range of about $0.15 \mathrm{~nm}$ at $T_{I}=50 \mathrm{eV}$, but the line centre depends only weakly on plasma conditions [16]. For the present experiments, a wavelength of $\lambda_{0}=529.06 \mathrm{~nm}$ is taken as reference value in equation (7). In case of $\mathrm{CX}$ with $\mathrm{C}^{5+}$, a reference value was obtained for practical purposes from the passive radiation at $\lambda_{0}=494.54 \mathrm{~nm}$ at the innermost observation channel. Since the poloidal rotation usually vanishes near the plasma centre, this value is expected to reflect the non-shifted emission. Spurious lineshifts due to cross-section effects [17] are neglected in first approximation since the energy dependence of the CX cross-sections for the Li-beam is much weaker than for a typical H-heating beam [13]. The sightlines are almost parallel to the poloidal velocity component as shown in figure 1 . At the present observation angle of $4^{\circ}$ to the poloidal plane, a toroidal velocity component has only a $7 \%$ projection on the direction of observation. In consequence, the measured lineshift can be attributed as a good approximation fully to the poloidal rotation.

In figure 3, the effect of the poloidal rotation is seen as a red-shift of spectra with regard to the reference wavelength. Figure 6 shows radial profiles of carbon poloidal velocities calculated from the measured lineshift (equation (7)) and the diamagnetic velocity of $\mathrm{C}^{6+}$ calculated from the impurity pressure profile (see below). Carbon ions rotate with velocities up to $35 \mathrm{~km} \mathrm{~s}^{-1}$ in counter ion diamagnetic direction. Poloidal rotation is located in a narrow shear layer at the plasma edge. Both species of carbon observed at different wavelengths yield

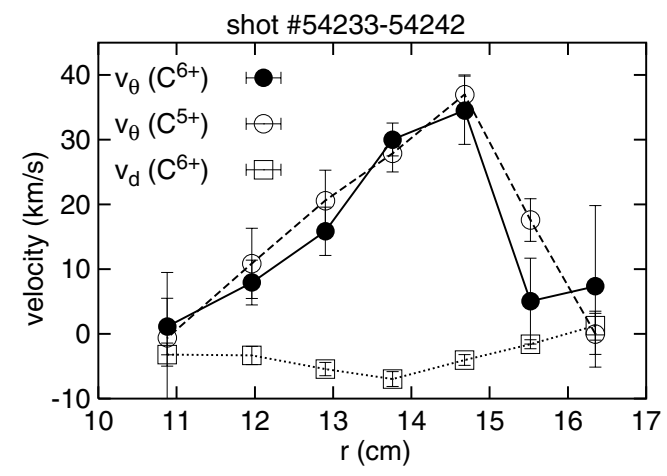

Figure 6. Poloidal rotation velocity $v_{\theta}$ measured by the Li-beam for $\mathrm{C}^{6+}$ and $\mathrm{C}^{5+}$. Also shown is the calculated diamagnetic velocity $v_{d}$ of the $C^{6+}$ ions according to equation (9). 
quite similar velocity profiles, which supports our confidence into the measurements. We note that in spite of the finite spectral resolution of $50 \mathrm{pm}$, a maximum precision of the measured lineshift of $5 \mathrm{pm}$ has been achieved with a total averaging time of $0.75 \mathrm{~s}$, which corresponds to an uncertainty in velocity of $\pm 2.5 \mathrm{~km} \mathrm{~s}^{-1}$.

The radial electric field is usually determined by a simplified radial force balance equation, in which friction, viscosity and inertia effects are neglected. Because of the complicated stellarator geometry, it is convenient to refer to the component $E_{\perp}$ perpendicular to $v_{\theta}$ and to the magnetic flux surface. For that case the force balance reads:

$$
E_{\perp}=\frac{1}{n_{I} Z_{I} e} \partial_{\perp}\left(n_{I} T_{I}\right)-v_{\theta} B_{\phi}+v_{\phi} B_{\theta},
$$

where $B_{\phi}$ and $B_{\theta}$ are the toroidal and poloidal components of the magnetic induction, respectively. We can express $\partial_{\perp}=\cos ^{-1} \alpha \partial / \partial z$ in equation (8), where $\alpha=23^{\circ}$ denotes the angle between the Li beam axis $z$ and the direction perpendicular to the magnetic flux surface. Since in W7-AS generally holds $B_{\theta} v_{\phi} \ll B_{\phi} v_{\theta}$, the term $v_{\phi} B_{\theta}$ can be neglected in the following [18]. This is due to $B_{\theta} / B_{\phi}<5 \%$ and viscous damping of the toroidal motion by collisions between passing and trapped particles. High values of up to $v_{\phi} \approx 65 \mathrm{~km} \mathrm{~s}^{-1}$ were observed in W7-AS only near the plasma centre and in case of unbalanced NBI. In conclusion, for the present measurements $v_{\phi}$ has no significant influence on the determination of $E_{\perp}$ in the edge region. Following equation (8), $E_{\perp}$ reaches values of up to $-950 \mathrm{~V} \mathrm{~cm}^{-1}$ at $r=14.7 \mathrm{~cm}$. This large negative value of $E_{\perp}$ at the edge is in general agreement with previous measurements employing a diagnostic hydrogen beam [18]. Rewriting equation (8), we obtain

$$
v_{\theta} \simeq \frac{E_{\perp}}{B_{\phi}}-\frac{1}{Z_{I} e n_{I} B_{\phi}} \partial_{\perp}\left(n_{I} T_{i}\right) .
$$

The first term in equation (9) is the contribution of the $E \times B$ drift and the second term is the diamagnetic drift $v_{d}$. The latter reaches up to $-6.8 \mathrm{~km} \mathrm{~s}^{-1}$ at $r=13.8 \mathrm{~cm}$ (figure 6). In consequence, the large positive poloidal velocity of $\mathrm{C}^{5+}$ and $\mathrm{C}^{6+}$ ions is attributed to the $E \times B$ drift.

Comparing $E_{\perp}$ with theory or other measurements, it has to be considered that-especially in stellarators $-E_{\perp}$ is a local quantity which is not constant on a magnetic flux surface. Since the electric potential $\Phi$ is usually assumed to be constant on a magnetic flux surface, the magnitude of $E_{\perp}$ depends on $(r, \phi, \theta)$ of the respective position via the distance between the flux surfaces. An effective radial electric field $E_{r}$ is defined as

$$
E_{r}=-\frac{\partial \Phi}{\partial r}
$$

where $r$ is the effective minor radius as above. It is related with $E_{\perp}$ by $E_{r}=E_{\perp} \cos \alpha \partial z / \partial r$, which yields

$$
E_{r} \simeq \frac{\partial z}{\partial r}\left\{\frac{1}{n_{I} Z_{I} e} \frac{\partial\left(n_{I} T_{I}\right)}{\partial z}-v_{\theta} B_{\phi} \cos \alpha\right\} .
$$

In the region of compressed flux surfaces, where the Li-beam enters the plasma, $\partial z / \partial r$ is about 0.7 . Figure 7 shows the resulting radial electric field as obtained for $C^{5+}$ and $C^{6+}$. Since no $\mathrm{C}^{5+}$ density could be determined, the term $n_{I}^{-1} \partial n_{I} / \partial z$ in equation (11) was neglected for $\mathrm{C}^{5+}$. For $\mathrm{C}^{6+}$ this term contributes to $E_{r}$ over the whole plasma radius with less than $35 \mathrm{~V} \mathrm{~cm}^{-1}$ and is therefore only a minor correction. Also shown in figure 7 is the calculation of $E_{r}$ carried out with the neoclassical transport code DKES, where $E_{r}$ is determined from the calculated ambipolar fluxes [19]. Good agreement of measurement and calculation is found.

Figure 8 shows a comparison of Li beam data with electric field measurements by passive $\mathrm{B}^{3+}$ radiation [18]. Due to insufficient accuracy in the spatial unfolding process of the $\mathrm{B}^{3+}$ 


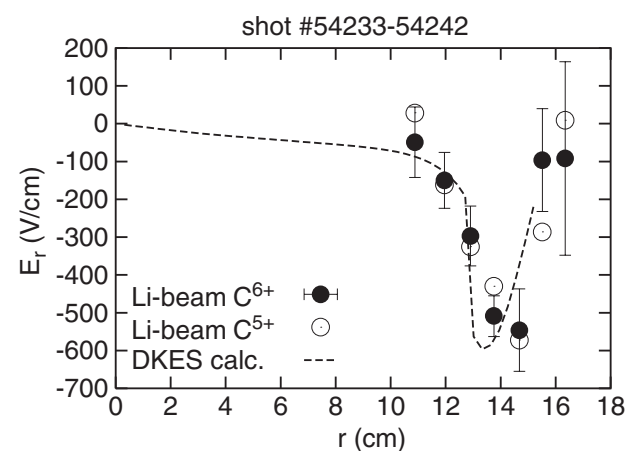

Figure 7. Radial electric field measured by $\mathrm{CX}$ with $\mathrm{Li}$ beam according equation $(11)(\bullet, \mathrm{O})$ and neoclassical calculation by DKES (- - -).

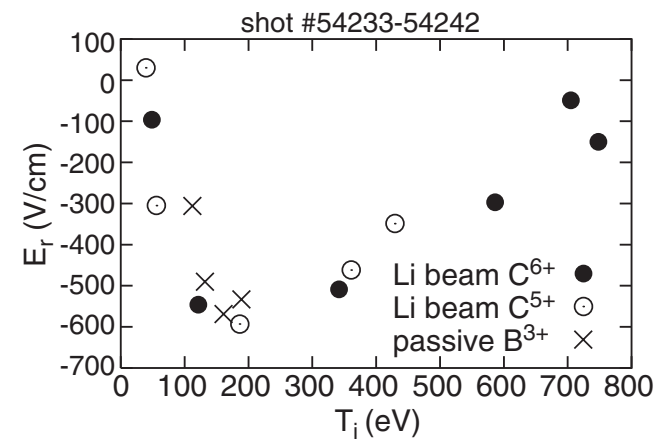

Figure 8. Radial electric field measured by $\mathrm{CX}$ with $\mathrm{Li}$ beam and passive $\mathrm{B}^{3+}$ radiation.

data, $E_{r}$ is plotted against $T_{i}(r)$ instead of $r$. The absolute values of $E_{r}$ agree very well within both diagnostics.

\section{Summary and conclusion}

This work has shown the high-energy Li beam to be a powerful diagnostic for the plasma edge and gradient region, with densities below $10^{20} \mathrm{~m}^{-3}$. Beam emission spectroscopy allows the determination of the electron density, whereas CX spectroscopy delivers-for sufficiently high carbon concentration - carbon ion density, temperature and poloidal rotation. From these measured quantities the radial electric field is determined in a straightforward manner. The resulting radial profile has high accuracy which allows detailed comparisons with neoclassical predictions.

Plasma parameters measured by the $\mathrm{Li}$ beam are combined measurements on the same coordinate. Such data is particularly valuable since a simultanuous measurement of these quantities usually involves several diagnostics with unavoidable geometric uncertainties. The high spatial resolution of the Li beam allows these measurements in steep edge gradients with an adequate precision, which is difficult to achieve with any other particle beam.

The fundamental role of the radial electric field in the reduction of neoclassical and anomalous transport $[19,20]$ underlines the importance of this work for magnetically confined high-temperature plasmas. 


\section{Acknowledgments}

H Ehmler would like to thank E Wolfrum, H Maassberg and H Wobig for fruitful discussion.

\section{References}

[1] Isler R C 1994 Plasma Phys. Control. Fusion 36171

[2] Ivanov A A et al 2000 Rev. Sci. Instrum. 713728

[3] McCormick K et al 1985 Rev. Sci. Instrum. 561063

[4] McCormick K et al 1999 Fusion Eng. Design 34125

[5] Schweinzer J et al 1992 Plasma Phys. Control. Fusion 341173

[6] Schorn R P et al 1991 Appl. Phys. B 5271

[7] Schorn R P et al 1992 Nucl. Fusion 32351

[8] Fiedler S et al 1999 J. Nucl. Mater. 2661279

[9] Brandenburg R et al 1999 Fusion Technol. 36289

[10] Renner H et al 1989 Plasma Phys. Control. Fusion 311579

[11] Kick M et al 1999 Plasma Phys. Control. Fusion 41549

[12] Kreter et al 2002 Proc. 29th EPS Controlled Fusion and Plasma Physics (Montreux, 2002) (Europhys. Conf. Abstracts) P-5.033

[13] Cornelius K R et al 2000 J. Phys. B: At. Mol. Opt. Phys. 332017

Wolfrum E 2000 Private communication

[14] Ehmler H et al 2002 Plasma Phys. Control. Fusion 441411

[15] Ehmler H et al 2002 Proc. 29th EPS Controlled Fusion and Plasma Physics (Montreux, 2002) (Europhys. Conf. Abstracts) P-4.044

[16] Kreter A 2001 Report 3860 Forschungszentrum Jülich E-mail: zb-publikation@fz-juelich.de

[17] Fonck R J et al 1984 Phys. Rev. A 293288

[18] Baldzuhn J et al 1998 Plasma Phys. Control. Fusion 40967

[19] Maassberg H et al 1993 Phys. Fluids B 53627

[20] Burrell K H 1997 Phys. Plasmas 41499 